\title{
Roturas retinianas em retinocoroidite por toxoplasmose: série de casos
}

\author{
Retinal tears in toxoplasmic retinochoroiditis: case series
}

\author{
Daniel da Rocha Lucena ${ }^{1}$ \\ Jefferson Augusto Santana Ribeiro ${ }^{2}$ \\ David da Rocha Lucena ${ }^{3}$ \\ Anna Lívia Pozzi de Lucena ${ }^{4}$ \\ Rodrig'o Jorgé ${ }^{5}$
}

Trabalho realizado na Faculdade de Medicina de Ribeirão Preto da Universidade de São Paulo - USP - Ribeirão Preto (SP) - Brasil.

${ }^{1}$ Médico contratado do Setor de Retina e Vítreo do Hospital das Clínicas da Faculdade de Medicina da Universidade de São Paulo - USP - Ribeirão Preto (SP) - Brasil. ${ }^{2}$ Pós-graduando nível doutorado Faculdade de Medicina da USP - Ribeirão Preto (SP) - Brasil.

${ }^{3}$ Pós-graduando nível mestrado Faculdade de Medicina da USP - Ribeirão Preto (SP) - Brasil.

${ }^{4}$ Aluna do $6^{\circ}$ ano do curso de medicina da Faculdade de Medicina da USP - Ribeirão Preto (SP) - Brasil.

${ }^{5}$ Docente do Departamento de Oftalmologia, Otorrinolaringologia e Cirurgia de Cabeça e Pescoço, Faculdade de Medicina da USP - Ribeirão Preto (SP) - Brasil.

Endereço para correspondência: Daniel da Rocha Lucena. Avenida Bandeirantes, 3.900 - $12^{\circ}$ andar Hospital das Clínicas - Campus - Ribeirão Preto (SP) CEP 14049-900

E-mail: drlucena@hcrp.fmrp.usp.br drlucena@terra.com.br

Recebido para publicação em 07.01.2009

Última versão recebida em 25.05.2009

Aprovação em 03.06.2009

\section{RESUMO}

Trata-se de série retrospectiva de 10 pacientes com rotura retiniana e retinocoroidite por toxoplasmose atendidos no Hospital das Clínicas da Faculdade de Medicina de Ribeirão Preto, de janeiro de 2007 a abril de 2008, com objetivo de avaliar a relação entre lesões de retinocoroidite e a ocorrência de rotura retiniana. Foram utilizados teste de Fisher e quiquadrado com nível de significância $p<0,05$. Oito casos (80\%) apresentaram descolamento de retina. Doze roturas foram identificadas, localizando-se principalmente na periferia temporal superior (6 casos, $50 \%$ ). Não foi observada relação estatisticamente significativa entre localização da rotura e da cicatriz coriorretiniana considerando a distribuição em cinco quadrantes $(\mathrm{p}=0,0828)$ ou em três zonas $(\mathrm{p}=0,2507)$. A ocorrência de roturas retinianas em pacientes com uveíte posterior pode estar relacionada ao descolamento precoce do vítreo posterior causado pelo processo inflamatório intraocular. Não foi observado neste estudo correlação entre a localização das roturas retinianas e as cicatrizes de coriorretinite, o que sugere um mecanismo não relacionado diretamente à cicatriz.

Descritores: Perfurações retinianas; Descolamento retiniano/cirurgia; Toxoplasmose; Coriorretinite; Uveíte; Humanos; Relatos de casos

\section{INTRODUÇÃO}

Descolamento de retina (DR) e roturas retinianas (RR) são complicações da toxoplasmose ocular ${ }^{(1)}$, não sendo muito frequente a ocorrência de descolamento de retina em pacientes com retinocoroidite toxoplásmica ${ }^{(2-3)}$.

Visto que ainda não é bem estabelecido o mecanismo pelo qual a retinocoroidite toxoplásmica leva às complicações citadas, realizou-se o presente estudo com o objetivo de avaliar a relação entre as lesões de toxoplasmose e as roturas retinianas.

\section{MÉTODOS}

Trata-se de estudo de casos retrospectivo envolvendo pacientes com roturas retinianas e retinocoroidite toxoplásmica atendidos no Hospital das Clínicas da Faculdade de Medicina de Ribeirão Preto, de janeiro de 2007 a abril de 2008.

Os pacientes incluídos tinham rotura retiniana com ou sem descolamento de retina e diagnóstico prévio de retinocoroidite por toxoplasmose baseado no aspecto das lesões e em achados sorológicos (IgG e/ou IgM positivas para toxoplasmose). 
Foram excluídos pacientes com outras causas de uveíte, como tuberculose, sífilis e sarcoidose, e pacientes cujo mapeamento de retina no exame inicial fosse impossível.

Os parâmetros principais de avaliação foram: localização da lesão cicatricial de retinocoroidite e localização da rotura retiniana. Para avaliar correlação entre estas duas variáveis foram utilizados teste de Fisher e qui-quadrado com nível de significância $\mathrm{p}<0,05$.

Também foram avaliadas as características das lesões de coriorretinite e roturas, bem como aspectos relacionados ao seu tratamento.

\section{RESULTADOS}

Foram incluídos 10 pacientes. Oito (80\%) apresentaram DR com rotura retiniana identificada, enquanto 2 (20\%) apresentaram somente rotura. A idade média foi 38 anos, variando de 14 a 69 anos. Sete (70\%) eram homens e 3 (30\%) mulheres.

Lesões de toxoplasmose foram bilaterais em 3 casos (30\%), e o número de cicatrizes foi uma em 5 pacientes $(50 \%)$ e duas nos outros 5 pacientes $(50 \%)$.

O tempo entre a ocorrência da retinocoroidite toxoplásmica e a rotura/DR foi igual a um mês em 2 pacientes (20\%), entre 5 e 12 meses em 3 (30\%), e maior que um ano em 3 casos (30\%), enquanto em 2 pacientes (20\%) o tempo não foi especificado (Tabela 1).

Sete pacientes $(70 \%)$ tinham recebido tratamento específico para toxoplasmose ocular, e os outros 3 (30\%), não. Cinco pacientes $(50 \%)$ foram tratados uma vez e 2 (20\%), mais de uma vez. O tempo decorrido entre o último tratamento e a ocorrência do DR/rotura foi um mês em 2 casos (20\%), entre 5 e 12 meses em 3 (30\%), e maior que um ano em 2 casos $(20 \%)$.

A acuidade visual corrigida (AV) no olho acometido pela rotura/DR na apresentação inicial foi percepção luminosa em 1 caso (10\%), movimento de mãos (MM) a 0,1 em $6(60 \%)$ e melhor que $0,1 \mathrm{em} 3$ casos (30\%). A AV na última consulta foi entre MM e 0,1 em 7 casos (70\%) e maior que 0,1 em outros 3 (30\%), tendo melhorado em 3 pacientes (30\%), piorado em outros $3(30 \%)$ e permanecido igual em 4 casos (40\%). O tempo médio de seguimento após a detecção da rotura/DR foi seis

\begin{tabular}{|c|c|c|c|c|}
\hline \multirow[b]{2}{*}{ Tratamento prévio } & \multicolumn{2}{|c|}{$\begin{array}{l}\text { Tempo } \\
\text { DR/RR* }\end{array}$} & \multicolumn{2}{|c|}{$\begin{array}{c}\text { № de } \\
\text { procedimentos }\end{array}$} \\
\hline & $<1$ ano & $>1$ ano & 1 & 2 \\
\hline Sim & 5 & 2 & 7 & 0 \\
\hline Não & 0 & 1 & 1 & 2 \\
\hline Total & 5 & 3 & 8 & 2 \\
\hline \multicolumn{5}{|c|}{$\begin{array}{l}\text { Tempo DR/RR= tempo entre a retinocoroidite e a ocorrência do descolamento de } \\
\text { retina/rotura retiniana; }{ }^{*}=\text { em dois casos não foi possível obter o tempo } D R / R R\end{array}$} \\
\hline
\end{tabular}

meses, com 8 casos (80\%) seguidos mais de seis meses, e 2 (20\%) menos de dois meses.

Com relação ao tratamento (Tabela 1 ), 8 pacientes $(80 \%)$ foram submetidos a um procedimento e $2(20 \%)$ a dois procedimentos [retinopexia convencional e, após, vitrectomia via pars plana (VVPP) com óleo de silicone]. VVPP com óleo de silicone foi realizada em 6 casos (60\%), retinopexia em 4 (40\%) e somente LASER em 2 pacientes (20\%). Na última avaliação, 1 paciente apresentou redescolamento de retina, e nenhum teve recidiva da retinocoroidite neste intervalo.

Roturas únicas foram identificadas em 8 pacientes $(80 \%)$ enquanto 2 (20\%) apresentaram duas roturas em um único olho. A localização das roturas ocorreu preferencialmente na região superior (Figura 1). Somente 3 roturas $(25 \%)$ se localizaram no mesmo quadrante da cicatriz, das quais apenas uma ocorreu adjacente à lesão cicatricial. Considerando cinco quadrantes no mapeamento de retina, a distribuição das roturas e cicatrizes coriorretinianas por quadrantes não mostrou associação estatisticamente significativa $(\mathrm{p}=0,0828)$. Analisando a distribuição das roturas e cicatrizes coriorretinianas por zonas (polo posterior, média periferia e periferia) (Tabela 2), também não se observou relação estatisticamente significativa entre essas duas variáveis $(\mathrm{p}=0,2507)$.

Oito roturas $(66,7 \%)$ foram caracterizadas como lesões em ferradura enquanto $4(33,3 \%)$ como buracos.

\section{DISCUSSÃO}

Pacientes com toxoplasmose ocular têm uma frequência aumentada de descolamento de retina ${ }^{(1)}$, sendo a uveíte, por si só, um fator de risco para o desenvolvimento de DR regmatogênico ${ }^{(4)}$.

Foi reportada a ocorrência de DR em $10 \%$ dos pacientes com toxoplasmose congênita ${ }^{(5)}$. Frequências de $6 \%$ de DR e de 5\% de roturas retinianas foram descritas por Bosch-Driessen et al. ${ }^{(1)}$, em uma série de 150 pacientes consecutivos com toxoplasmose ocular, taxas semelhantes aos 5\% e 3,5\% de DR em pacientes com toxoplasmose ocular presumida descritos por outros au-

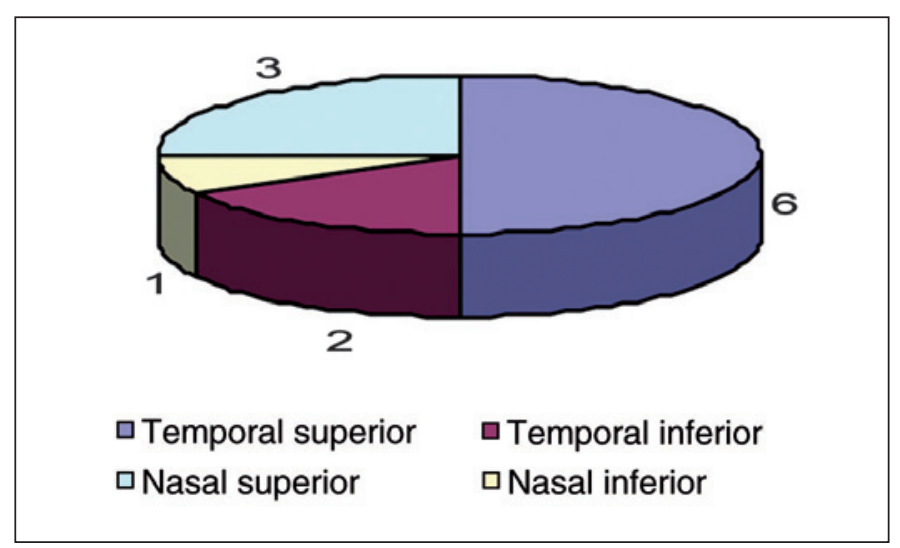

Figura 1 - Distribuição das roturas retinianas com relação à localização por quadrante em pacientes com retinocoroidite toxoplásmica 


\begin{tabular}{|c|c|c|c|}
\hline \multirow[b]{2}{*}{ Zona da cicatriz } & \multicolumn{2}{|c|}{ Zona da rotura } & \multirow[b]{2}{*}{ Total } \\
\hline & II & $\overline{\text { III }}$ & \\
\hline I & 1 & 6 & 7 \\
\hline II & 0 & 1 & 1 \\
\hline III & 0 & 2 & 2 \\
\hline Total & 1 & 9 & 10 \\
\hline
\end{tabular}

tores $^{(4,6)}$. Uma das possibilidades para explicar o DR em pacientes com uveíte é o descolamento do vítreo posterior, que ocorre precocemente $^{(7)}$, e por sua vez é causado pelo processo inflamatório intraocular ${ }^{(3)}$.

As roturas retinianas na retinocoroidite toxoplásmica foram observadas sob duas formas características ${ }^{(3)}$ : roturas em ferradura e roturas na margem de cicatrizes de retinocoroidite. No presente trabalho, $8(66,7 \%)$ roturas possuíam aspecto em ferradura, e $4(33,3 \%)$ foram caracterizadas como buracos.

Com relação à localização da rotura, maior frequência é encontrada nos quadrantes temporais, principalmente o temporal superior, sendo menos frequentes no quadrante nasal inferior em pacientes sem descolamento de retina ${ }^{(8-9)}$. Estes dados estão em consonância com o verificado no presente trabalho. Correlacionando-se a localização das roturas com a localização da lesão coriorretiniana, em geral, as primeiras localizaram-se em quadrante diferente das segundas, com apenas 3 roturas $(25 \%)$ localizadas no mesmo quadrante da cicatriz, sendo uma destas adjacentes à cicatriz. Assim, não se observou relação direta entre a localização da rotura e a localização da cicatriz coriorretiniana, estando implicado como o fator causal da RR nos pacientes deste estudo em geral o descolamento do vítreo posterior secundário ao processo inflamatório prévio.

Retinopexia com explante de silicone se mostrou eficaz no tratamento do DR secundário à retinocoroidite toxoplásmica, sem afetar o curso da retinocoroidite ${ }^{(3)}$, embora o prognóstico visual do DR regmatogênico em uveíte seja ruim ${ }^{(4)}$. Os pacientes deste estudo que se apresentaram somente com rotura retiniana foram tratados com laserterapia cercando a lesão, enquanto VVPP com infusão de óleo de silicone foi o tratamento empregado na maioria dos casos com descolamento de retina (6 casos, 60\%). No período de seguimento, os indivíduos apresentaram evolução favorável, sem recidiva da retinocoroidite, ocorrendo melhora da acuidade visual, porém, em somente 3 pacientes ( $30 \%)$.

A ocorrência de roturas retinianas em pacientes com retinocoroidite toxoplásmica não se mostrou relacionada di- retamente à lesão coriorretiniana, podendo ser uma consequência do descolamento do vítreo posterior precoce na maioria dos casos, embora um pequeno número de pacientes tenha sido estudado. O exame da periferia retiniana em pacientes com toxoplasmose ocular é fundamental para a detecção de roturas $\mathrm{e}$, consequentemente, seu tratamento precoce, visando evitar o descolamento de retina.

\section{ABSTRACT}

This study is a retrospective case series aiming to evaluate the relation between toxoplasmic retinochoroiditis scars and the occurrence of retinal tears. Ten patients with retinal tear and toxoplasmic retinochoroiditis examined at the School of Medicine of Ribeirão Preto Clinics Hospital, between January 2007 and April 2008, were included. Fisher test and qui-square test with significance level of $\mathrm{p}<0.05$ were used. Eight cases (80\%) had retinal detachment. Twelve tears were found and localized mostly in the temporal superior periphery ( 6 cases, $50 \%$ ). No statistically significant association between retinal tear and chorioretinal scar localization was observed considering five retinal quadrants $(\mathrm{p}=0.0828)$ or three zones $(\mathrm{p}=0.2507)$. The occurrence of retinal tears in patients with uveitis may be related to early posterior vitreous detachment caused by the intraocular inflammatory process. No correlation was observed between the localization of retinal tears and chorioretinal scars in this study, which suggests a causative factor not directly related to the presence of a scar.

Keywords: Retinal perforations; Retinal detachment/surgery; Toxoplasmosis; Chorioretinitis; Uveitis; Human; Case reports

\section{REFERÊNCIAS}

1. Bosch-Driessen LH, Karimi S, Stilma JS, Rothova A. Retinal detachment in ocular toxoplasmosis. Ophthalmology. 2000;107(1):36-40.

2. Atmaca LS, Simsek T, Batioglu F. Clinical features and prognosis in ocular toxoplasmosis. Jpn J Ophthalmol. 2004;48(4):386-91.

3. Lucier AC. Retinal detachment associated with ocular toxoplasmosis. Trans Am Acad Ophthalmol Otolaryngol. 1974;78:882-9.

4. Kerkhoff FT, Lamberts QJ, van den Biesen PR, Rothova A. Rhegmatogenous retinal detachment and uveitis. Ophthalmology. 2003;110(2):427-31.

5. Mets MB, Holfels E, Boyer KM, Swisher CN, Roizen N, Stein L, et al. Eye manifestations of congenital toxoplasmosis. Am J Ophthalmol. 1996;122(3): 309-24.

6. Friedmann CT, Knox DL. Variations in recurrent active toxoplasmic retinochoroiditis. Arch Ophthalmol. 1969;81(4):481-93.

7. Hirokawa H, Takahashi M, Trempe CL. Vitreous changes in peripheral uveitis. Arch Ophthalmol. 1985;103(11):1704-7.

8. Combs JL, Welch RB. Retinal breaks without detachment: natural history, management and long term follow-up. Trans Am Ophthalmol Soc. 1982;80:64-97.

9. Hyams SW, Neumann E. Peripheral retina in myopia. With particular reference to retinal breaks. Br J Ophthalmol. 1969;53(5):300-6. 